\title{
Abdominal CT radiation dose optimization at Siriraj Hospital (phase II)
}

Piyaporn Apisarnthanarak, M.D.

Suchanya Hongpinyo, M.D.

Krittya Saysivanon, B.Sc.

Chulaluck Boonma, B.Sc.

Sureerat Janpanich, $R N$.

Preeyanuch Ketkan, RN.

Kobkun Muangsomboon, M.D.

Wanwarang Teerasamit, M.D.

Sopa Pongpornsup, M.D.

Pairash Saiviroonporn, Ph.D.

From Department of Radiology, Faculty of Medicine Siriraj Hospital, Mahidol University, Bangkok, Thailand.

Address correspondence to P.A. (e-mail: punpae159@gmail.com)

Received 28 April 2020; revised 12 December 2020; accepted 14 December 2020 doi:10.46475/aseanjr.v21i3.81

\section{Abstract}

Objective: To compare radiation dose, radiologists' satisfaction, and image noise between the standard dose abdominal CT currently performed at our hospital and the new automatic tube current modulation (ATCM) low dose abdominal CT, using various parameters $(0 \%, 10 \%, 20 \%$, and $30 \%)$ of the Adaptive Statistical Iterative Reconstruction (ASiR).

Materials and Methods: We prospectively performed the ATCM low dose abdominal CT in 111 participants who had prior standard dose CT for comparison. The ATCM low dose CT images were post processed with 4 parameters $(0 \%, 10 \%, 20 \%$ and $30 \%)$ of $\mathrm{ASiR}$ on a CT workstation. The volume 
CT dose index (CTDIvol) of the ATCM low dose and the standard dose CT were compared. Four experienced abdominal radiologists independently assessed the quality of the ATCM low dose CT with the aforementioned ASiR parameters using a 5-point-scale satisfaction score $(1=$ unacceptable, $2=$ poor, $3=$ average, $4=$ good, and $5=$ excellent image quality) by using the prior standard dose CT as a reference of an excellent image quality (5). Each reader selected the preferred ASiR parameter for each participant. The image noise of the liver and the aorta in all 5 techniques (1 prior standard dose and 4 current ATCM low dose techniques) was measured. The correlation between the image quality vs the participants' body mass index (BMI) and waist circumferences were analyzed.

Results: The mean CTDIvol of the ATCM low dose CT was significantly lower than of the standard dose CT $(7.29 \pm 0.20$ vs $11.28 \pm 0.23 \mathrm{mGy}, \mathrm{p}<0.001)$. The mean satisfaction score for the ATCM low dose CT with $0 \%, 10 \%, 20 \%$ and $30 \%$ ASiR were 4.14, 4.16, 4.17, and 4.26, respectively with the ranges of 3 to 5 in all techniques. The preferred ASiR parameters of each participant randomly selected by each reader were varied, depending on the readers' opinions. The mean image noise of the aorta on the standard dose CT and the ATCM low dose CT with 0\%, $10 \%, 20 \%$, and $30 \%$ ASiR was $30.69,36.60,34.05,31.43$, and 29.09 , respectively, while the mean image noise of the liver was 24.96, 29.90, 27.86, 25.66, and 23.68, respectively. There was a correlation between the image quality (satisfaction score and image noise) vs the participants' BMI and waist circumferences.

Conclusion: The ATCM low dose CT received acceptable radiologists' satisfaction with significant radiation dose reduction. The increment of ASiR was helpful in reducing the image noise and had a tendency to increase the radiologists' satisfaction score.

Keywords: Abdominal computed tomography, Abdominal CT, Automatic tube current modulation, ATCM, Radiation dose optimization, Iterative reconstruction, IR, Adaptive statistical iterative reconstruction, ASiR. 


\section{Introduction}

Nowadays, many new computed tomography (CT) techniques have been proposed for the improved image quality by providing thin slice collimation and fast rotation time. In spite of getting better image resolution and ability to achieve a dynamic study, it comes with the high radiation exposure, which is considered one of the potential risks of cancer[1]. There have been many proposed techniques for radiation dose optimization, such as minimizing the number of CT acquisitions and area coverage as necessary, decreasing the tube current and the peak kilovoltage[2,3]. Automatic tube current modulation (ATCM) enables automatic adjustment of the tube current according to the size and density characteristics of the body part being scanned. It is one of the most accepted techniques for radiation dose optimization. It reduces radiation dose with an acceptable image quality as well as a fixed tube current (FTC) technique.[4-6].

However, the radiation dose reduction will inevitably increase the image noise, degrade the image quality, and disturb the image interpretation. Over the past decade, the CT vendors offered many techniques for optimizing the image quality of the low dose CT scan. One well-accepted reconstruction technique was iterative reconstruction (IR) which helped reduce the image noise compared with the conventional filtered back projection (FBP) reconstruction technique and could help diminish the radiation dose of 30-50\%[7-9].

Our prior study on the abdominal CT radiation dose optimization[10] was prospectively performed in 119 participants by using the FTC technique (30\% reduction of standard tube current: from 400 to be $260 \mathrm{~mA}$ on 64-slice CT scanner, and from 340 to be $210 \mathrm{~mA}$ on 256 -slice CT scanner). We applied the new de-noising IR technique (Adaptive Statistical Iterative Reconstruction, ASiR) with various parameters $(0 \%, 10 \%, 20 \%$, and $30 \% \mathrm{ASiR})$ by post-processing on a CT workstation to improve the image quality of the low dose CT. The result of the study provided significant radiation dose reduction with an acceptable image quality. 
This current phase II study was a prospective study on abdominal CT radiation dose optimization using the ATCM technique, another attractive technique for CT radiation dose optimization. The received images were also post-processed on a CT workstation with 4 parameters of $\mathrm{ASiR}(0 \%, 10 \%, 20 \%$, and $30 \%)$. The purposes of the current study were to compare radiation dose, radiologists' satisfaction, and the image noise between the standard dose abdominal CT currently performed at our hospital and the new ATCM low dose abdominal CT, using various parameters of $\mathrm{ASiR}$ techniques.

\section{Materials and methods}

\section{Study Designs and Participants}

This study was a prospective, single-centered study performed at a 2,200-bed university hospital in central Thailand. This study was approved by our institutional review board with informed consents from all included participants.

All participants were aged over 18 years old who were scheduled for contrast enhanced abdominal CT examinations at our department during January 2019. They had available prior standard dose contrast enhanced abdominal CT within 180 days for comparison. In total, one hundred and eleven participants met the criteria and were recruited as our study population. The demographic data of each participant including gender, age, body mass index (BMI), and waist circumference were recorded by one of our investigators $(\mathrm{SH})$.

\section{CT Techniques}

\section{Standard Dose Abdominal CT}

The prior standard dose abdominal CT of our participants was routinely performed by four General Electric (GE) CT scanners including three 64-slice scanners (one LightSpeed VCT and two Discovery CT750 High Definition, GE Healthcare, Milwaukee, WI, USA) and one 256-slice scanner (Revolution CT, GE healthcare, Milwaukee, WI, USA). The CT protocol of each participant was selected for 
a proper number of CT acquisitions and area coverage. All participants were advised to hold their breath during the scan. The scan coverage included at least the upper abdominal area. The slice collimation was $1.25 \mathrm{~mm}$ (reconstructed at $7.0 \mathrm{~mm}$ ) for all scanners. There were varieties on the administration of oral and rectal contrasts according to each participant's appropriate protocol. All participants underwent precontrast and postcontrast studies, before and after a bolus intravenous injection of the nonionic iodinated contrast agent $(2 \mathrm{~mL}$ per $\mathrm{kg}$ body weight), followed by 20 mLof water via a power injector at a rate of $3 \mathrm{~mL} /$ second. Each participant had at least a portovenous phase with an 80-second delay for postcontrast study. An additional arterial phase at 35 to 40-second delay or delayed phase at 5 to 10 -minute delay was obtained in some participants as necessary. The peak kilovoltage was fixed at $120 \mathrm{kVp}$ for all scanners. The fixed tube current based on our standard protocol was $400 \mathrm{~mA}$ and $340 \mathrm{~mA}$ for 64-slice and 256-slice CT scanners, respectively. The rotation time was 0.5 seconds for all scanners. The pitch was 1.375:1 and 0.992:1 for 64-slice and 256-slice CT scanners, respectively. All images were reconstructed with the standard FBP techniques and sent to the Picture Archiving and Communication System (PACS) for subsequent reviews.

\section{ATCM Low Dose Abdominal CT}

The ATCM low dose abdominal CT was performed by three GE CT scanners including two 64-slice scanners (Discovery CT750 High Definition, GE Healthcare, Milwaukee, WI, USA) and one 256-slice scanner (Revolution CT, GE healthcare, Milwaukee, WI, USA). Our old-fashioned 64-slice CT scanner (LightSpeed VCT, GE healthcare, Milwaukee, WI, USA) did not have the IR de-noising technique for improving the image quality; therefore, it was not included in the performance of the low dose abdominal CT. The CT scanners for the standard and low dose CT of each participant were not necessarily the same scanners. The CT protocol of each participant was selected for a proper number of CT acquisitions and area coverage (at least covering the upper abdominal area). The scan techniques were the same as described in the prior standard dose abdominal CT section except for the tube current which was automatic adjusted by the CT scanners between 150-270 mA with a fixed noise index of 18, according to the size and density characteristics of each participant's abdomen (ATCM technique). 
The IR technique specific for our GE CT scanners (Adaptive Statistical Iterative Reconstruction, ASiR) was applied by blending with the conventional FBP on low dose portovenous phase images by post-processing on a CT workstation by using the 4 parameters of ASiR: 0\% ASiR (with 100\% FBP), 10\% ASiR (with 90\% FBP), 20\% ASiR (with 80\% FBP) and 30\% ASiR (with 70\% FBP). With these reconstruction techniques, four sets of low dose portovenous CT images were created and sent to PACS for subsequent reviews. We chose to study only on the portovenous phase because most abdominal organs had homogeneous enhancement on this phase. It was easy for radiologists to evaluate the CT image quality.

For a parameter of radiation dose comparison, we selected the volume CT dose index (CTDIvol) instead of dose length product (DLP). The DLP would depend on the length of scan which varied in the participants due to the difference in area coverage and the number of CT acquisitions.

The details of CT scanners, study dates, and CTDIvol of each participant's prior standard dose abdominal CT and the current ATCM low dose abdominal CT were recorded by one of our investigators $(\mathrm{SH})$. The time interval between the two studies was calculated.

\section{Image Quality Assessment}

For qualitative image quality assessment, four board-certified, abdominal radiologists (PA, KM, WT, and SP with 23, 23, 17, and 17 years of experience in abdominal CT evaluation) independently reviewed one set of the standard dose portovenous abdominal CT images and 4 sets of ATCM low dose portovenous abdominal CT images with $0 \%, 10 \%, 20 \%$, and $30 \%$ ASiR of each participant. All readers were blinded to the percentage of the applied ASiR. They graded the image quality of each low dose CT set by using a 5-point-scale satisfaction score on a visual scale as follows: 
1: Unacceptable image quality, unable to interpret

2: Poor image quality, interfering with interpretation

3: Average image quality, possible interpretation

4: Good image quality

5: Excellent image quality

The satisfaction score was given by using each participant's prior standard dose CT images as a reference of an excellent image quality (5). The satisfaction scores of 3 to 5 were acceptable for CT interpretation. Each reader selected the preferred ASiR parameter from 4 sets of ATCM low dose abdominal CT for each participant.

For quantitative image quality assessment of the abdominal CT, the image noise of the aorta and the liver was measured on one set of the standard dose CT images and other 4 sets of the ATCM low dose CT images by one of our investigators $(\mathrm{SH})$ on a CT workstation (Advantage workstation AW 4.6, GE healthcare, Milwaukee, WI, USA). The image noise was measured by drawing a circular region of interests (ROIs) at 4 locations (one aortic and 3 hepatic regions) on a $1.25-\mathrm{mm}$ slice portovenous image at the same locations and levels of these 5 image sets. For the image noise of the aorta, the ROI was drawn at least $1 / 3$ area of the aortic lumen (range $68-116 \mathrm{~mm} 2$, mean $98.48 \mathrm{~mm} 2 \pm 6.40 \mathrm{~mm} 2$ ) at the most central part to avoid calcified plaque at the aortic wall. For the image noise of the liver, 3 hepatic ROIs (range 74-108 $\mathrm{mm} 2$, mean $100.25 \mathrm{~mm} 2 \pm 2.69 \mathrm{~mm} 2$ ) were routinely applied on the left lobe, the anterior right lobe, and the posterior right lobes (Figure 1). In patients with prior hepatic surgery, the ROIs were placed in three different locations in the remaining hepatic areas (Figure 2). The hepatic ROIs were placed at the homogenous enhancing hepatic areas avoiding vessels, bile ducts, hepatic lesions, calcifications and surgical materials. The mean image noise of each liver was calculated from these 3 hepatic ROIs of the image noise. 


\section{Statistical Analysis}

The demographic data of participants, CT scanners, time interval between CT studies, the image quality (satisfaction scores, readers' preferred ASiR parameters, image noise) and CTDIvol of the ATCM low dose and the standard dose CT were presented as number (\%), mean (standard deviation, SD), median, and range. Paired t-test was used to compare mean CTDIvol between the standard dose CT and the low dose CT. Multivariate analysis with Bonferroni adjustment for a pairwise comparison was applied to compare satisfaction scores, the mean image noise of the aorta and the liver among different ASiR parameters. Spearman correlation was used to evaluate the relationship between the satisfaction score vs the participants' BMI and waist circumferences. The Pearson's correlation was used to evaluate the relationship between the image noise vs the participants' BMI and waist circumferences.

All statistical data analyses were performed by using PASW 18.0 (SPSS Inc., Chicago, IL, USA). A 2-sided p-value of less than or equal to 0.05 was considered as a statistical significance.

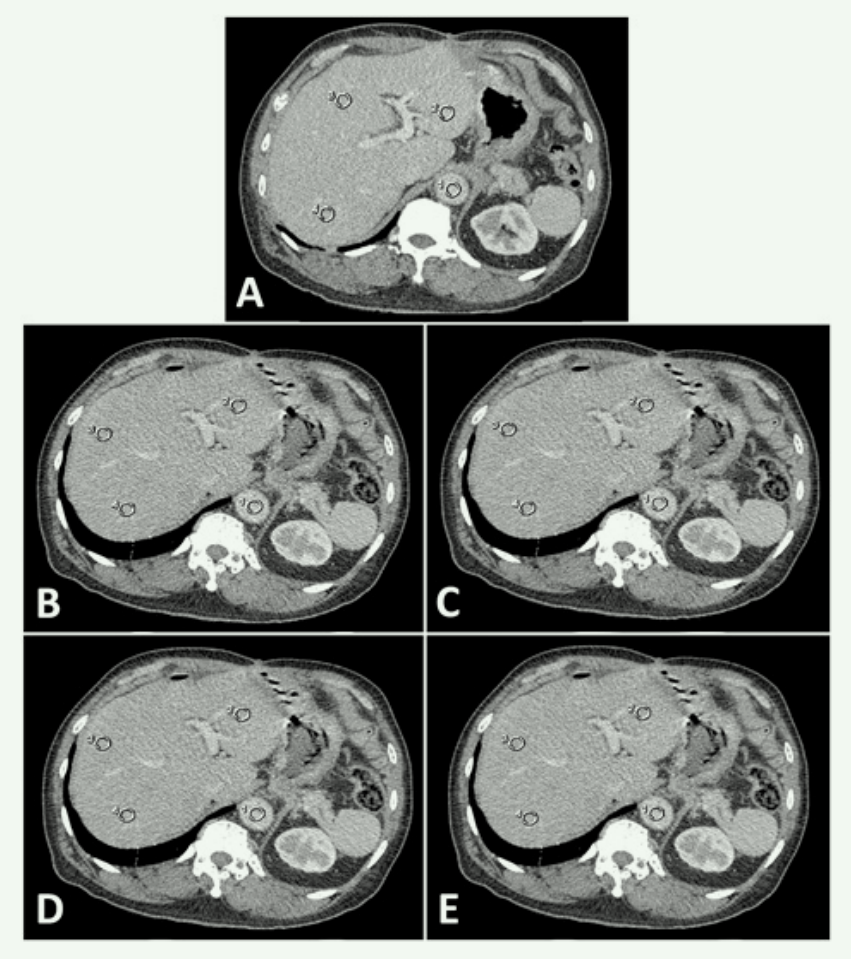

Figure 1. The image noise measurement of the aorta (1 ROI) and the liver (3 ROIs at the left lobe, the anterior right lobe and the posterior right lobe) on the 5 image sets (A-E)

A: Prior standard dose abdominal CT

$B-E$ : The current ATCM low dose abdominal CT with 0\% ASiR (B), 10\% ASiR (C), $20 \% \mathrm{ASiR}(\mathrm{D})$ and $30 \% \mathrm{ASiR}(\mathrm{E})$

The ROIs were positioned at the same locations and levels for all 5 image sets. 


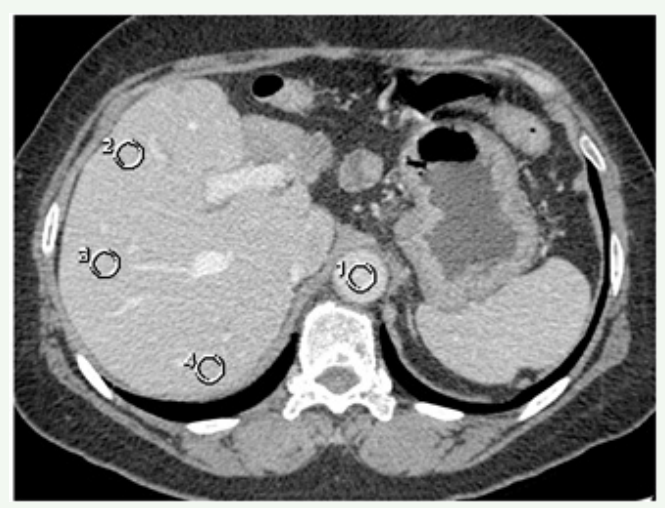

Figure 2. In case of post left hepatectomy, the 3 hepatic ROIs were placed in three different locations in the remaining right hepatic area.

\section{Results}

\section{Participants}

One hundred and eleven participants in this study included 63 (56.8\%) men and $48(43.2 \%)$ women. The mean age (SD) of the participants at the time of the ATCM low dose CT scan was 61.4 (12.9) years with the range of 21-95 years.

The mean BMI (SD) was $23.44(3.89) \mathrm{kg} / \mathrm{m}^{2}$ (range $16.01-34.29 \mathrm{~kg} / \mathrm{m}^{2}$, median $23.05 \mathrm{~kg} / \mathrm{m}^{2}$ ). With respect to BMI category, number of participants with underweight $\left(B M I<18.5 \mathrm{~kg} / \mathrm{m}^{2}\right)$, normal weight $\left(\right.$ BMI $\left.18.5-24.9 \mathrm{~kg} / \mathrm{m}^{2}\right)$, overweight (BMI $\left.25.0-29.9 \mathrm{~kg} / \mathrm{m}^{2}\right)$ and obesity $\left(\mathrm{BMI} \geq 30.0 \mathrm{~kg} / \mathrm{m}^{2}\right)$ were $11(9.9 \%), 63(56.8 \%)$, $30(27.0 \%)$, and $7(6.3 \%)$, respectively. The mean waist circumference (SD) was $84.4(9.2) \mathrm{cm}$ (range $66-113 \mathrm{~cm}$, median $83 \mathrm{~cm}$ ).

\section{CT Techniques}

The standard dose abdominal CT of 72 (64.9\%) and 39 (35.1\%) participants were performed by 64 -slice and 256-slice scanners, respectively. The ATCM low dose abdominal CT of $65(58.6 \%)$ and $46(41.4 \%)$ participants were performed by 64-slice and 256-slice scanners, respectively. The time interval between the two studies ranged from 13 to 178 days (median 132 days). 
The mean CTDIvol (SD) of the ATCM low dose CT was significantly lower than that of standard dose CT, $7.29(0.20)$ vs $11.28(0.23) \mathrm{mGy}(\mathrm{p}<0.001)$.

\section{Image Quality Assessment}

For qualitative image quality assessment, the satisfaction score of the ATCM low dose abdominal CT with $4 \mathrm{ASiR}$ parameters graded by 4 readers ranged from 3 to 5 , which were all acceptable for CT interpretation. The mean satisfaction scores of the ATCM low dose abdominal CT with 4 ASiR parameters were summarized in Table 1. The preferred ASiR parameters applied to the low dose CT of each participant was randomly selected by each reader, displayed in Table 2 .

For quantitative image quality assessment, the image noise of the aorta and the liver on the standard dose CT and the ATCM low dose CT with 4 ASiR parameters was summarized in Table 3 and 4 (Figure 3 and 4). As expected, the ATCM low dose CT had a higher image noise of the aorta and the liver than the standard dose $\mathrm{CT}$. After applying the ASiR technique, the image noise on the low dose CT images decreased. The higher percentage of ASiR employed, the lower image noise of the aorta and the liver was obtained.

There was a correlation between the image quality (satisfaction score and image noise) vs the participants' BMI and waist circumferences. Patients with high BMI and large waist circumferences received lower satisfaction scores and had more image noises on their CT images. The correlation of the image quality vs BMI was stronger than vs waist circumference (Table 5 and 6 ). 
Table 1. The mean satisfaction scores of the ATCM low dose abdominal CT with $0 \%$, $10 \%, 20 \%$, and $30 \%$ ASiR parameter graded by 4 readers.

\begin{tabular}{lllll} 
& \multicolumn{4}{c}{ Mean Satisfaction Scores (SD) of ATCM Low Dose Abdominal CT } \\
\cline { 2 - 5 } & 0\% ASiR & $\mathbf{1 0 \%}$ ASiR & 20\% ASiR & 30\% ASiR \\
\hline Reader 1 & $4.53(0.52)$ & $4.53(0.54)$ & $4.50(0.52)$ & $4.50(0.54)$ \\
Reader 2 & $3.24(0.43)$ & $3.33(0.47)$ & $3.39(0.49)$ & $3.74(0.44)$ \\
Reader 3 & $4.19(0.72)$ & $4.18(0.72)$ & $4.19(0.71)$ & $4.23(0.71)$ \\
Reader 4 & $4.61(0.49)$ & $4.60(0.49)$ & $4.60(0.49)$ & $4.56(0.50)$ \\
All readers & $4.14(0.40)$ & $4.16(0.41)$ & $4.17(0.40)$ & $4.26(0.39)$ \\
\hline
\end{tabular}

Note: There were significant statistical differences between

$0 \%$ ASiR vs 30\% ASiR ( $p<0.001)$; 95\%CI (0.062, 0.163)

$10 \%$ ASiR vs 30\% ASiR ( $p<0.001) ; 95 \% C I(0.043,0.146)$

$20 \%$ ASiR vs 30\% ASiR ( $p<0.001) ; 95 \% C I(0.038,0.133)$

Table 2. The preferred ASiR parameters applied to the ATCM low dose abdominal CT selected by 4 readers.

\begin{tabular}{lccccc}
\hline & \multicolumn{3}{c}{ Number of The Preferred ASiR Parameter (\%) } & \multirow{2}{*}{ Total } \\
\cline { 2 - 5 } & 0\% ASiR & 10\% ASiR & 20\% ASiR & 30\% ASiR & A \\
\hline Reader 1 & $48(43.2)$ & $33(29.7)$ & $21(18.9)$ & $9(8.1)$ & $111(100.0)$ \\
Reader 2 & $7(6.3)$ & $9(8.1)$ & $19(17.1)$ & $76(68.5)$ & $111(100.0)$ \\
Reader 3 & $3(2.7)$ & $15(13.5)$ & $28(25.2)$ & $65(58.6)$ & $111(100.0)$ \\
Reader 4 & $68(61.3)$ & $26(23.4)$ & $9(8.1)$ & $8(7.2)$ & $111(100.0)$
\end{tabular}


Table 3. The image noise of the aorta and the liver on standard dose CT and ATCM low dose CT with 4 different ASiR parameters.

\begin{tabular}{lccccc}
\hline & $\begin{array}{c}\text { Standard } \\
\text { Dose CT }\end{array}$ & 0\% ASiR & 10\% ASiR & 20\% ASiR & 30\% ASiR \\
\cline { 3 - 6 } & & & & & \\
Aorta & & & & \\
Mean (SD) & $30.69(6.39)$ & $36.60(6.33)$ & $34.05(6.06)$ & $31.43(5.70)$ & $29.09(5.43)$ \\
\hline Min & 17.43 & 22.84 & 21.56 & 19.80 & 18.28 \\
Max & 47.50 & 56.20 & 53.67 & 50.47 & 47.93 \\
\hline Liver & & & & & \\
Mean (SD) & $24.96(5.62)$ & $29.90(5.63)$ & $27.86(5.08)$ & $25.66(4.65)$ & $23.68(4.35)$ \\
\hline Min & 13.90 & 17.18 & 15.73 & 14.64 & 13.47 \\
Max & 41.68 & 46.48 & 43.33 & 40.49 & 37.70 \\
\hline
\end{tabular}

Table 4. The differences of the mean image noise of the aorta and the liver on the $A T C M$ low dose CT with 4 different ASiR parameters compared to the standard dose CT.

\begin{tabular}{|c|c|c|c|}
\hline & $\begin{array}{c}\text { Differences (SD) of Mean Image Noise of Low } \\
\text { Dose CT Compared to Standard Dose CT }\end{array}$ & p-Value & $95 \%$ CI \\
\hline \multicolumn{4}{|l|}{ Aorta } \\
\hline $0 \%$ ASiR & $5.91(0.57)$ & $<0.001$ & $4.28,7.56$ \\
\hline $10 \% \mathrm{ASiR}$ & $3.36(0.56)$ & $<0.001$ & $1.75,4.98$ \\
\hline $20 \% \mathrm{ASiR}$ & $0.74(0.58)$ & 1.000 & $-0.91,2.39$ \\
\hline $30 \% \mathrm{ASiR}$ & $-1.60(0.57)$ & 0.059 & $-3.22,0.03$ \\
\hline \multicolumn{4}{|l|}{ Liver } \\
\hline $0 \%$ ASiR & $4.94(0.34)$ & $<0.001$ & $3.96,5.92$ \\
\hline $10 \%$ ASiR & $2.90(0.33)$ & $<0.001$ & $1.95,3.86$ \\
\hline $20 \% \mathrm{ASiR}$ & $0.70(0.34)$ & 0.400 & $-0.26,1.66$ \\
\hline 30\% ASiR & $-1.28(0.34)$ & 0.003 & $-2.26,-0.30$ \\
\hline
\end{tabular}




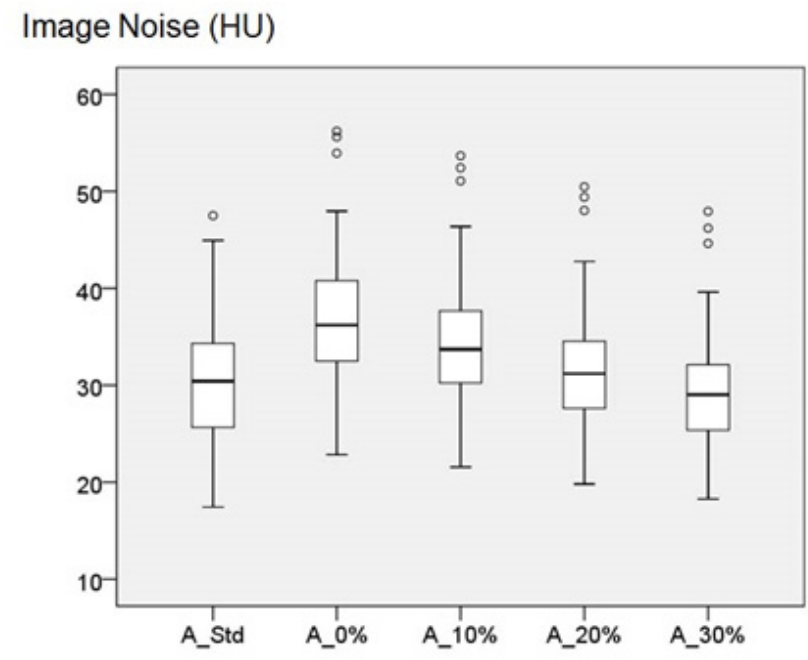

Figure 3. The graph shows the mean image noise (range) of the aorta on the standard dose CT (A_std) and the ATCM low dose CT with 0\% ASiR (A_0\%), 10\% ASiR (A_10\%), 20\% ASiR (A_20\%) and 30\% ASiR (A_30\%).

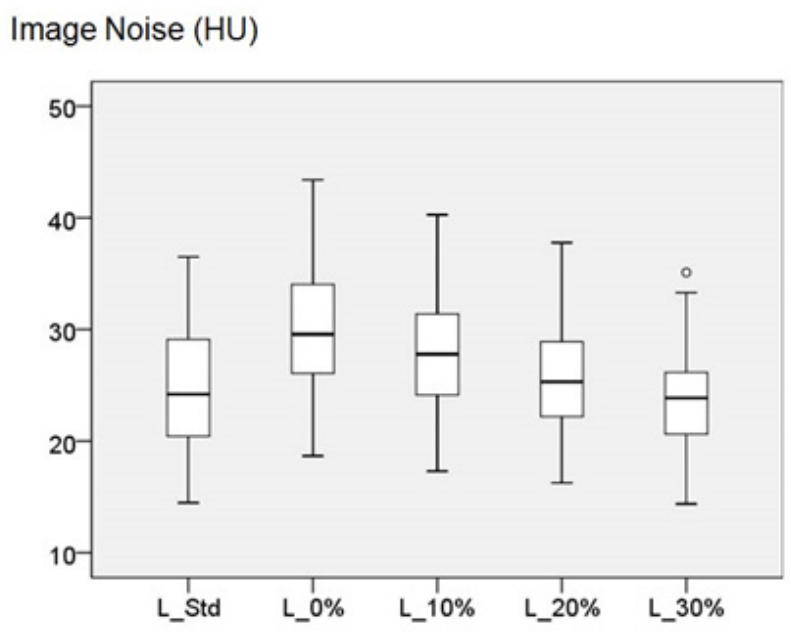

Figure 4. The graph shows the mean image noise (range) of the liver on the standard dose CT (L_std) and the ATCM low dose CT with 0\% ASiR (L_0\%), 10\% ASiR (L_10\%), 20\% ASiR (L_20\%) and 30\% ASiR (L_30\%). 
Table 5. The correlation between satisfaction score vs BMI and waist circumference on the standard dose CT and the low dose CT with different ASiR parameters.

\begin{tabular}{lcccc} 
& \multicolumn{2}{c}{ BMI } & \multicolumn{2}{c}{ Waist Circumference } \\
\hline & $\mathbf{R}$ & $\mathbf{p}$-Value & $\mathbf{R}$ & p-Value \\
\hline $0 \%$ ASIR & -0.333 & $<0.001$ & -0.222 & 0.19 \\
$10 \%$ ASIR & -0.364 & $<0.001$ & -0.229 & 0.16 \\
$20 \%$ ASIR & -0326 & $<0.001$ & -0.204 & 0.32 \\
$30 \%$ ASIR & -0.313 & $=0.001$ & -0.192 & 0.43
\end{tabular}

Table 6. The correlation between the image noise of the aorta and the liver vs BMI and waist circumference on the standard dose CT and the low dose CT with different ASiR parameters.

\begin{tabular}{lcccc}
\hline \multicolumn{2}{c}{ BMI } & & \multicolumn{2}{c}{ Waist Circumference } \\
\hline \multicolumn{1}{c}{$\mathbf{R}$} & $\mathbf{p}$-Value & $\mathbf{R}$ & p-Value \\
\hline Aorta & & & & \\
Standard & 0.633 & $<0.001$ & 0.599 & $<0.001$ \\
$0 \%$ ASIR & 0.516 & $<0.001$ & 0.475 & $<0.001$ \\
\hline $10 \%$ ASIR & 0.504 & $<0.001$ & 0.475 & $<0.001$ \\
$20 \%$ ASIR & 0.471 & $<0.001$ & 0.459 & $<0.001$ \\
$30 \%$ ASIR & 0.470 & $<0.001$ & 0.480 & $<0.001$
\end{tabular}

\section{Liver}

\begin{tabular}{lllll} 
Standard & 0.737 & $<0.001$ & 0.646 & $<0.001$ \\
\hline $0 \%$ ASIR & 0.729 & $<0.001$ & 0.610 & $<0.001$ \\
\hline $10 \%$ ASIR & 0.690 & $<0.001$ & 0.584 & $<0.001$ \\
\hline $20 \%$ ASIR & 0.700 & $<0.001$ & 0.594 & $<0.001$ \\
\hline $30 \%$ ASIR & 0.671 & $<0.001$ & 0.585 & $<0.001$
\end{tabular}




\section{Discussion}

With the worldwide concern about radiation safety, there have been several novel CT reconstruction techniques to enhance the image quality, allowing the radiologists to continue reducing the CT radiation dose for the sake of patient's safety while still accomplishing the satisfactory image quality. The IR is one of the new image reconstruction techniques that have been developed in the past decade. Previous studies assessing the imaging quality obtained from the low dose CT with the IR techniques reported that the IR technique helped improve the image quality by decreasing the image noise and provided a comparable image quality as the standard dose CT[11-13].

At our hospital, we used ASiR (IR technique specific for our GE CT scanners) to blend with the conventional FBP technique to optimize the image quality of the low dose CT. As a result of our study, the ATCM low dose abdominal CT significantly reduced the radiation dose compared to the standard dose abdominal CT. The image quality of all low dose techniques was acceptable for interpretation, although the image noise was significantly increased in $0 \%$ ASiR and $10 \%$ ASiR image sets. When applying $20 \%$ and $30 \%$ ASiR to the ATCM low dose abdominal CT images, there was no significant difference of the image noise of the aorta and the liver compared to the standard dose CT. Our results were similar to several prior studies[10-16].

Our study showed that the mean satisfaction score had a tendency to increase when the higher percentage ASiR applied. The 30\% ASiR image set showed a significantly higher satisfaction score compared to other low dose image sets. For the preferred image set, there were different opinions between 4 readers. Two readers preferred the lower percentage of ASiR, while the other two readers preferred the higher percentage of ASiR. We assumed that the images with the high percentage of ASiR provided smooth image appearances with less sharp borders. This was the reported major drawback of the IR technique[11,13]. Some of our readers were possibly familiar with a relatively noisy image with sharp borders derived from the conventional FBP technique. 
Our study showed the correlation between the image quality (satisfaction score and image noise) vs the participants' BMI and waist circumferences. Patients with high BMI or large waist circumferences received lower satisfaction scores and had more image noises on their CT images. After applying ASiR, it helped improve the image quality in patients with high BMI and large waist circumference. Shaqdan et al.[17] supported the result of our study. They reported that IR provided a significantly higher image quality (less image noise and higher contrast-to-noise ratio) compared to the FBP reconstruction technique in obese patients.

This current study was designed to diminish the limitations of our phase I study [10]. Reviewers in this current study were blinded to the percentage of ASiR applied to ATCM low dose CT images; and the time interval between the standard dose and the ATCM low dose CT was limited within 180 days. We found that either FTC (phase I study) or ATCM (the current study) low dose CT with ASiR provided an acceptable image quality and significant radiation dose reduction compared with standard dose CT. From our experience, the FTC technique is simple and easy to be performed, while ATCM is more complicated, requires technicians with more expertise. A direct comparison between FTC and ATCM should be further studied for more accurate information.

With the attempt to reduce the radiation dose for the sake of patient's safety, the image quality is inevitably decreased although many novel reconstruction techniques are applied. Radiologists should realize the importance of radiation optimization and open their mind to adopt low dose CT images with an acceptable image quality. Radiologists are the key people to balance the amount of radiation dose reduction and the suitable image quality for accurate CT interpretation.

There were several limitations of our study. First, there were variables in our CT scanners. Although they were all GE scanners, most were 64-slice scanners and one was a 256-slice scanner. Of which, some CT parameters (i.e. $\mathrm{mA}$ and pitch) were not the same. Inherent differences in scanners could affect the results of the study. Plus, the CT scanners for the standard and the low dose CT of each participant were not necessarily the same scanners. Second, the time interval 
between the prior standard dose CT and the current low dose CT ranged from 13 to 178 days (median 132 days). Although shorter than 6 months, there could be any changes in participants' habitus or conditions that could affect the image quality. The new study with a shorter time interval should be designed. Third, the image noise was measured on a $1.25 \mathrm{~mm}$ slice portovenous image of each image set. Actually, the image noise should be measured by choosing 3-5 consecutive CT slices and the noise should be averaged for the statistical accuracy. Finally, our study focused only on the image quality (satisfaction score and image noise) of the low dose CT. We did not study diagnostic performances of the low dose CT. To evaluate the diagnostic performances between the low dose CT and the standard dose CT, these 2 studies should be performed on the same date and in almost the same acquisition phase. These will inevitably increase the radiation dose received by the participants.

In conclusion, the ATCM low dose CT with the tube current between 150 and 270 $\mathrm{mA}$ and a fixed noise index of 18 received acceptable radiologists' satisfaction with significant radiation dose reduction. The increment of ASiR technique was helpful in reducing the image noise and had a tendency to increase the radiologists' satisfaction score. 


\section{References}

1. Hall EJ, Brenner DJ. Cancer risks from diagnostic radiology: the impact of new epidemiological data. Br J Radiol 2012;85:e1316-7. doi: 10.1259/bjr/13739950.

2. Hara AK, Wellnitz CV, Paden RG, Pavlicek W, Sahani DV. Reducing body $\mathrm{CT}$ radiation dose: beyond just changing the numbers. AJR Am J Roentgenol 2013;201:33-40. doi: 10.2214/AJR.13.10556.

3. Tamm EP, Rong XJ, Cody DD, Ernst RD, Fitzgerald NE, Kundra V. Quality initiatives: CT radiation dose reduction: how to implement change without sacrificing diagnostic quality. Radiographic 2011;31:1823-32. doi: 10.1148/ rg.317115027.

4. Kalra MK, Maher MM, Toth TL, Schmidt B, Westerman BL, Morgan HT, et al. Techniques and applications of automatic tube current modulation for CT. Radiology 2004;233:649-57. doi: 10.1148/radiol.2333031150.

5. Kalra MK, Maher MM, Toth TL, Kamath RS, Halpern EF, Saini S. Comparison of Z-axis automatic tube current modulation technique with fixed tube current CT scanning of abdomen and pelvis. Radiology 2004;232:347-53. doi: 10.1148/ radiol.2322031304.

6. Lee S, Yoon SW, Yoo SM, Ji YG, Kim KA, Kim SH, et al. Comparison of image quality and radiation dose between combined automatic tube current modulation and fixed tube current technique in CT of abdomen and pelvis. Acta Radiol 2011;52:1101-6. doi: 10.1258/ar.2011.100295.

7. Patino M, Fuentes JM, Singh S, Hahn PF, Sahani DV. Iterative reconstruction techniques in abdominopelvic CT: Technical concepts and clinical implementation. AJR Am J Roentgenol 2015;205:W19-31. doi: 10.2214/ AJR.14.13402. 
8. Willemink MJ, de Jong PA, Leiner T, de Heer LM, Nievelstein RA, Budde RP, et al. Iterative reconstruction techniques for computed tomography Part 1: technical principles. Eur Radiol 2013;23:1623-31. doi: 10.1007/s00330-012$2765-\mathrm{y}$.

9. Geyer LL, Schoepf UJ, Meinel FG, Nance JW Jr, Bastarrika G, Leipsic JA, et al. State of the art: iterative CT reconstruction techniques. Radiology 2015;276: 339-57. doi: 10.1148/radiol.2015132766.

10. Apisarnthanrak P, Buranont C, Boonma C, Janpanich S, Suwatananonthakij T, Klinhom A, et al. Abdominal CT radiation dose optimization at Siriraj Hospital. ASEAN J Radiol 2020;21:28-43.

11. Mitsumori LM, Shuman WP, Busey JM, Kolokythas O, Koprowicz KM. Adaptive statistical iterative reconstruction versus filtered back projection in the same patient: 64 channel liver CT image quality and patient radiation dose. Eur Radiol 2012;22:138-43. doi: 10.1007/s00330-011-2186-3.

12. Prakash P, Kalra MK, Kambadakone AK, Pien H, Hsieh J, Blake MA, et al. Reducing abdominal CT radiation dose with adaptive statistical iterative reconstruction technique. Invest Radiol 2010;45:202-10. doi: 10.1097/RLI. ob013e3181dzfeec.

13. Sagara Y, Hara AK, Pavlicek W, Silva AC, Paden RG, Wu Q. Abdominal CT: comparison of low-dose CT with adaptive statistical iterative reconstruction and routine-dose CT with filtered back projection in 53 patients. AJR Am J Roentgenol 2010;195:713-9. doi: 10.2214/AJR.09.2989.

14. Willemink MJ, Takx RA, de Jong PA, Budde RP, Bleys RL, Das M, et al. Computed tomography radiation dose reduction: effect of different iterative reconstruction algorithms on image quality. J Comput Assist Tomogr 2014;38:815-23. doi: 10.1097/RCT.0000000000000128. 
15. Gervaise A, Osemont B, Louis M, Lecocq S, Teixeira P, Blum A. Standard dose versus low-dose abdominal and pelvic CT: comparison between filtered back projection versus adaptive iterative dose reduction 3D. Diagn Interv Imaging 2014;95:47-53. doi: 10.1016/j.diii.2013.05.005.

16. Singh S, Kalra MK, Shenoy-Bhangle AS, Saini A, Gervais DA, Westra SJ, et al. Radiation dose reduction with hybrid iterative reconstruction for pediatric CT. Radiology 2012;263:537-46. doi: 10.1148/radiol.12110268.

17. Shaqdan KW, Kambadakone AR, Hahn P, Sahani DV. Experience with iterative reconstruction techniques for abdominopelvic computed tomography in morbidly and super obese patients. J Comput Assist Tomogr 2018;42:124-32. doi: 10.1097/RCT.0000000000000656. 